\title{
Urolithin A Mitigates Cisplatin-Induced Nephrotoxicity by Inhibiting Renal Inflammation and Apoptosis in an Experimental Rat Mode|[\$
}

\author{
Melissa Guada, Raghu Ganugula, Manicka Vadhanam, and Majeti N.V. Ravi Kumar \\ Department of Pharmaceutical Sciences, College of Pharmacy, Texas A\&M University, College Station, Texas (M.G., R.G., \\ M.N.V.R.K.); and Department of Medicine, University of Louisville, Louisville, Kentucky (M.V.)
}

Received April 25, 2017; accepted July 31, 2017

\begin{abstract}
Cumulative kidney toxicity associated with cisplatin is severe and there is no clear consensus on the therapeutic management of the same. The pathogenesis involves activation of inflammatory and apoptotic pathways; therefore, regulating these pathways offers protection. Given the anti-inflammatory and antioxidant effects of urolithin $\mathrm{A}$, a gut microbial metabolite of ellagic acid, our aim was to explore the potential use of urolithin $\mathrm{A}$ in the prevention of cisplatin-induced nephrotoxicity in an experimental rat model. For this purpose, animals received a single intraperitoneal dose of cisplatin $(5 \mathrm{mg} / \mathrm{kg}$ body weight). Six hours prior to cisplatin administration, rats were orally treated with either ellagic acid or urolithin A $(50 \mathrm{mg} / \mathrm{kg}$ body weight), followed by a daily dose of these compounds during the next 5 days. At the end, plasma and kidneys were collected for analysis. Cisplatin-induced kidney damage was
\end{abstract}

revealed by a significant rise in the plasma creatinine levels accompanied by significant morphologic changes in tubules, $\mathrm{T}$ cell $\mathrm{Ig}$ and mucin domain-containing protein-1, ionized calcium-binding adapter molecule 1 , as well as a marked increase in the number of apoptotic cells localized in tubules. Cisplatin also reduced nitric oxide synthase 3 and nuclear factor kappa-light-chain-enhancer of activated $B$ cells resulting in regulation of various inflammatory cytokines. Urolithin $A$ effectively attenuated cisplatin-induced kidney damage and showed significantly greater effect than its precursor ellagic acid on preserving the normal kidney architecture by downregulating the proinflammatory cytokines. In summary, urolithin A mitigates cisplatin-induced nephrotoxicity in rats by modulation of the inflammatory cascade and inhibition of the proapoptotic pathway.

\section{Introduction}

Increasing the efficacy of cytotoxic drugs without compromising nontarget tissue safety is a critical issue in the field of oncology, and is still a major therapeutic approach to treating both localized and metastasized cancers (Mathijssen et al., 2014). Platinum-based drugs such as cisplatin (CIS) are widely used in the treatment of human and veterinary neoplasms' alike (Barabas et al., 2008; Dilruba and Kalayda, 2016). However, their severe side effects and resistance to the treatment (Barabas et al., 2008; Apps et al., 2015; Dilruba and Kalayda, 2016) limit clinical success. Among the undesirable effects, patient morbidity and mortality are negatively impacted by nephrotoxicity (Yao et al., 2007; Bhat et al., 2015). Over the years, a range of strategies such as reformulating CIS, pharmacogenomics-based personalized dose regimens, use of adjuvants, etc., have been applied in clinical practice to mitigate CIS-induced nephrotoxicity (Pabla and Dong, 2008; Boulikas, 2009; Ciccolini et al., 2011). However, kidney-related toxicity

The authors declare no conflicts of interest.

M.G. and R.G. contributed equally to this work.

https://doi.org/10.1124/jpet.117.242420.

S This article has supplemental material available at jpet.aspetjournals.org. remains an issue in patients' health care and thus their quality of life.

The plant polyphenols are extensively investigated as adjuvants for their ability to sensitize drug-resistant cancer cells to the chemotherapy as well as their ability to protect nontarget tissues from damage by their antioxidant and antiinflammatory properties (Koyner et al., 2008; Lecumberri et al., 2013). Previously, we reported that ellagic acid (EA), a natural compound found in certain nuts, fruits, and vegetables, can protect kidney against cyclosporine-induced nephrotoxicity in the experimental rat model (Sharma et al., 2007; Sonaje et al., 2007). Similarly, the protective effect of EA against CIS-induced kidney injury has also been experimentally investigated (Ateşşahín et al., 2007; Al-Kharusi et al., 2013; El-Garhy et al., 2014). The beneficial effects reported are due to the reduction of oxidative stress and inhibition of proinflammatory and proapoptotic pathways, e.g., monocyte chemoattractant protein-1, nuclear factor kappa-light-chainenhancer of activated B cells (NF- $\kappa \mathrm{B})$, tumor necrosis factor- $\alpha$, inducible nitric oxide synthase, and nitric oxide (Ateșşahín et al., 2007). Recent reports suggest that most of the pharmacological properties associated with EA may be attributed to its gut microbial metabolites (urolithins), which are found in

ABBREVIATIONS: CIS, cisplatin; EA, ellagic acid; Iba1, ionized calcium-binding adapter molecule 1; IL, interleukin; NF- $\kappa$ B, nuclear factor kappalight-chain-enhancer of activated B cells; NOS3, nitric oxide synthase 3; PAS, periodic acid-Schiff; TIM-1, T cell Ig and mucin domain-containing protein-1; UA, urolithin A. 
higher levels in circulation compared with their precursor, after consumption of food rich in EA content (Kang et al., 2016; Ryu et al., 2016; Saha et al., 2016; Yuan et al., 2016). There is significant interest in therapeutic applications of urolithin A (UA), such as in treating prostate, bladder, and colon cancers as well as cardiovascular conditions (Zhou et al., 2016; Liberal et al., 2017; Tang et al., 2017). Furthermore, the availability of chemical synthesis for UA makes it an attractive candidate for investigating therapeutic applications.

To our knowledge, this is the first study to examine the potential of UA treatment in the prevention of CIS-induced nephrotoxicity in a rat model using the precursor compound EA as a reference. To provide insight into the mechanism of renal protection associated with UA, kidney injury was examined by histology, renal function, and apoptosis, as well as different molecular mediators, including $\mathrm{T}$ cell $\mathrm{Ig}$ and mucin domain-containing protein (TIM) $-1, \mathrm{NF}-\kappa \mathrm{B}$, nitric oxide synthase 3 (NOS3), ionized calcium-binding adapter molecule 1 (Iba1), and various inflammatory cytokines.

\section{Materials and Methods}

Reagents. CIS was obtained from Shaanxi Sciphar Hi-Tech Industry Co., Ltd. (China); UA (AGN-PC-0LQZ0Q) was obtained from Angene International Limited (London United Kingdom); EA was obtained from Sigma-Aldrich (Milwaukee, WI); and anti-TIM-1 antibody [EPR6383(2)] N-terminal ab190696 was obtained from ABCAM (Cambridge, MA). Anti- $\beta$-actin loading control monoclonal antibody

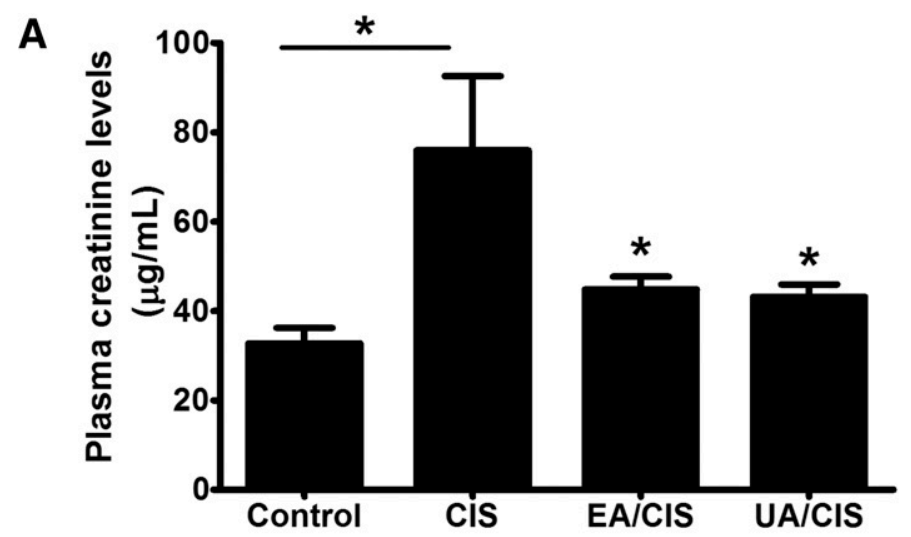

B

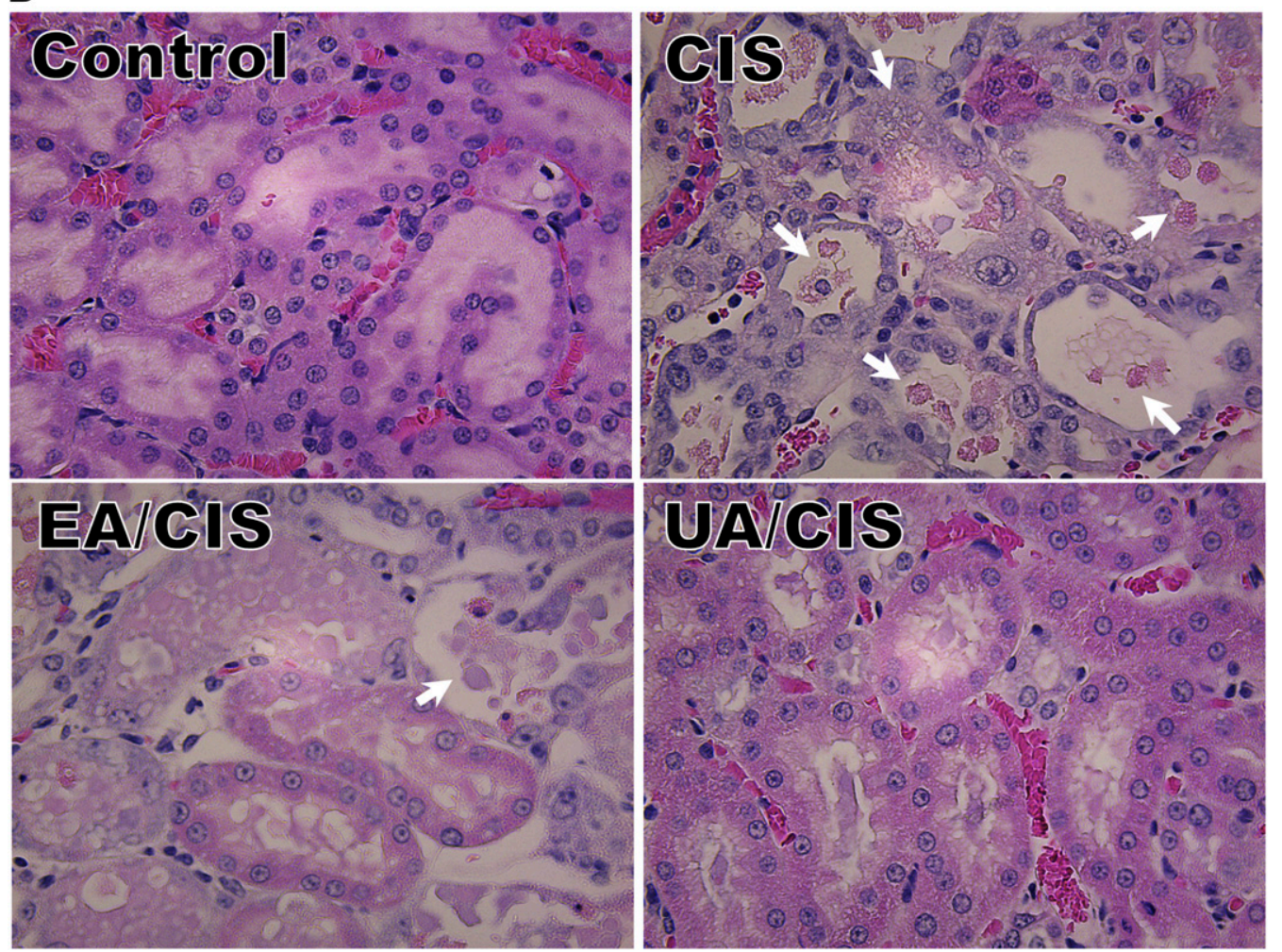

Fig. 1. Preventive effect of UA and EA on CIS-induced renal dysfunction and histopathological damage. (A) Plasma creatinine levels and (B) histologic changes in kidney tissue were observed by hematoxylin and eosin staining from each group $(n=6)$ on day 6 after CIS administration to rats (arrows indicate tubular injury). Images were captured using a bright-field microscope at original magnification, $40 \times{ }^{*} P<0.05$ compared with the CIS group; Mann-Whitney $U$ test. 
(BA3R), goat anti-rabbit and goat anti-mouse IgG $(\mathrm{H}+\mathrm{L})$ secondary antibodies, horseradish peroxidase and Coomassie Protein Assay Reagent were obtained from Thermo Fisher Scientific (Rockford, IL). NOS3 (C-20): sc-654 and NF- $\kappa$ B p65 (F-6): sc-8008 were obtained from Santa Cruz Biotechnology, Inc. (Dallas, TX) and anti-Iba1 antibody was obtained from Wako Chemicals USA, Inc. (Richmond, VA).

Animals and Experimental Design. Male Sprague-Dawley rats (200-300 g) were purchased from Harlan (Houston, TX). All experimental procedures were approved by the Texas A\&M University Institutional Animal Care and Use Committee (protocol number IACUC 2014-0106). Animals were maintained in a standard housing environment and had free access to food and water during the study. After acclimatization, rats were randomly divided into four groups $(n=6)$ as follows: group I, negative control; group II, positive control (CIS); groups III and IV, EA/CIS and UA/CIS, respectively. On the first day of the experiment, groups II, III, and IV received a single intraperitoneal dose of CIS ( $5 \mathrm{mg} / \mathrm{kg}$ body weight) dissolved in saline. Six hours prior to CIS administration, groups III and IV were orally treated with either EA or UA (50 mg/kg body weight) suspended in $0.5 \%$ (w/v) carboxymethyl cellulose aqueous solution, followed by a daily dose of these compounds during the next 5 days. Animal weights were recorded throughout the experiment. On day 7 , the animals were euthanized by $\mathrm{CO}_{2}$ asphyxiation followed by cervical dislocation. Blood samples were taken by heart puncture, collected into $\mathrm{K}_{2}$ EDTA-coated tubes and centrifuged at $956 \mathrm{~g} / 4^{\circ} \mathrm{C}$ for 30 minutes for plasma separation (stored at $-80^{\circ} \mathrm{C}$ until analysis). After perfusion with saline via the circulatory system, both kidneys were collected, weighed, and longitudinally cut into two sections; one section was used for histology (stored in 10\% formalin) and the other section was used for molecular analysis (frozen immediately in liquid nitrogen and stored at $-80^{\circ} \mathrm{C}$ ).

Biochemical Analysis. Creatinine levels in plasma were measured using a quantitative enzyme-linked immunosorbent assay kit specific to rat following the manufacturer's protocol (MyBioSource, Inc., San Diego, CA). Plasma samples were diluted at a ratio of 1:4 using the sample dilution buffer provided by the manufacturer.

Histologic Examination. Formalin-fixed kidneys were embedded in paraffin and sectioned into $4 \mu \mathrm{m}$ thickness. These sections were stained with H\&E and periodic acid-Schiff (PAS) reagents for histologic examination. Tissue sections were evaluated using a bright-field microscopy at $40 \times$ original magnification (3000-LED Microscope; ACCU-SCOPE Commack, NY). Five representative microscopy images taken from different rats within the same group were analyzed using the ImageJ software to measure the PAS-positive area and the results were expressed as the average percentage of the total area.

Western Blot. Kidneys were homogenized in phosphate-buffered saline at a final tissue concentration of $10 \%(\mathrm{w} / \mathrm{v})$ and centrifuged at $20817 \mathrm{~g} / 4^{\circ} \mathrm{C}$ for 30 minutes. Supernatant was collected and protein concentration was determined using the Coomassie Protein Assay (Thermo Scientific). Approximately $20 \mu \mathrm{g}$ of protein was loaded into a $4 \%-15 \%$ one-dimensional SDS-polyacrylamide gel and subjected to electrophoresis. Next, the proteins were transferred to a nitrocellulose membrane and unreacted sites were blocked with 5\% nonfat milk for 1 hour at room temperature. The membranes were incubated with the primary antibody (TIM-1 at 1:10000 and $\beta$-actin at 1:2000) overnight at $4^{\circ} \mathrm{C}$, washed with a mixture of Tris-buffered saline and Tween 20 , and then incubated with the appropriate secondary antibody for another 2 hours at room temperature. The chemiluminescent detection method was used to visualize protein signals. The band images were obtained by the ChemiDoc imaging system (Bio-Rad) and were analyzed by densitometry using the ImageLab software (Bio-Rad, Hercules, CA). $\beta$-Actin was used to normalize the protein loading for each sample.

Immunohistochemistry. Paraffin-kidney sections were heated at $60^{\circ} \mathrm{C}$ for 1 hour, and then washed twice with xylene for 10 minutes to ensure paraffin removal. The sections were rehydrated in serial ethanol dilutions $(100 \%, 95 \%$, and $70 \%)$. After washing the slides in water, antigen retrieval was achieved by boiling the tissue sections in sodium-citrate buffer (0.01 M, pH 6.0) three times for 5 minutes in a microwave oven. The slides were cooled down to room temperature, washed with phosphate-buffered saline, and then blocked and stained using a commercial kit for detecting primary antibodies (ImmunoCruz Rabbit ABC Staining System: sc-2018; Santa Cruz Biotechnology, Inc.). Kidney sections were incubated with primary antibodies specific

\section{A}
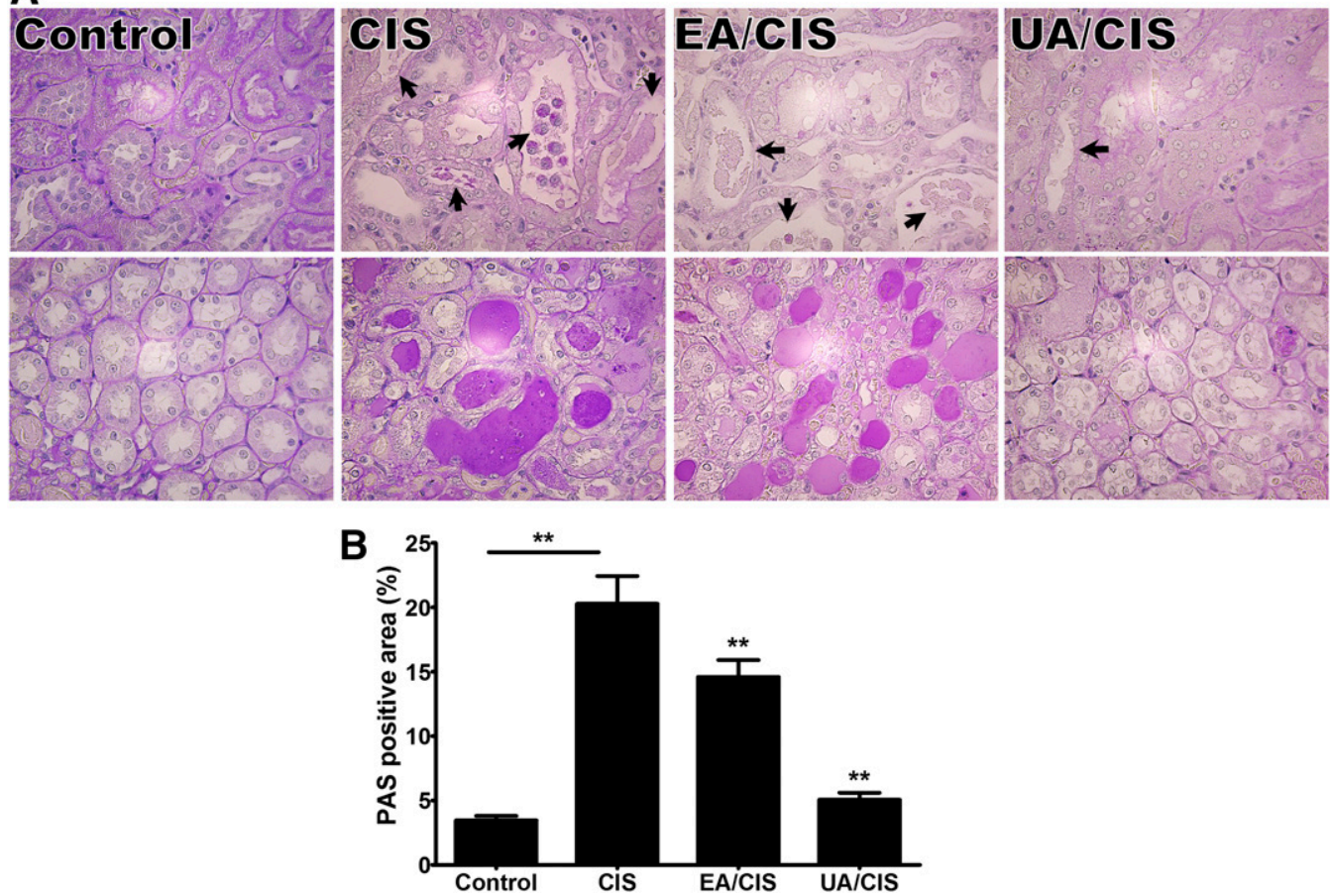

Fig. 2. Protective effect of UA, and to a lesser extent EA, on tubular morphology of kidneys injured by CIS exposure. (A) Kidney sections stained with PAS obtained from each group $(n=6)$ on day 6 after CIS administration to rats (arrows indicate tubular damage and magenta-colored spots represent PAS-positive area). Representative images are shown at original magnification, 40×. (B) Quantitative analysis of PAS-positive area reflecting the degree of tubular injury. ${ }^{*} P<0.01$ compared with the CIS group; Mann-Whitney $U$ test. 
to TIM-1 (1:200), NF- $\kappa$ B p65, NOS3, and Iba1 (1:100) overnight at $4^{\circ} \mathrm{C}$ and all other staining steps were performed according to the manufacturers' instructions. Tissue sections were counterstained with hematoxylin, dehydrated, and mounted for microscopy analysis. Images were taken with a bright-field microscope at the original magnification of $40 \times$ (3000-LED Microscope; ACCU-SCOPE).

Multiplex Immunoassay. The quantification of different cytokines in plasma and kidney homogenates were performed using the ProcartaPlex Immunoassay Kit specific to rat, based on magnetic beads technology (eBioscience, San Diego, CA), following the manufacturer's instructions. The cytokine concentrations on each sample were measured using a microplate reader (Bio-Plex 200 System; Bio$\mathrm{Rad})$ and the values obtained were normalized by the protein content in the case of kidney homogenates.

Immunofluorescence. Kidney sections were deparaffinized, rehydrated, and washed as previously described for immunohistochemistry staining. After antigen retrieval, tissue sections were stained with a terminal deoxynucleotidyl transferase dUTP nick end labeling reaction mixture using an in situ cell death detection kit (Roche, Mannheim, Germany). Slides were mounted using Vectashield Antifade Mounting Media containing 4',6-diamidino-2-phenylindole (Vector Laboratories, Burlingame, CA). Tissue sections were imaged using a confocal laser scanning microscope (Zeiss LSM 780, Carl Zeiss Microscopy GmbH, Jena, Germany) at $40 \times$ original magnification. Terminal deoxynucleotidyl transferase dUTP nick end labeling positive cells were quantified using the ImageJ software and the results were expressed as average of at least 22 images for each group.

Statistical Analysis. The data are expressed as mean values \pm S.D. Statistical analysis was performed using GraphPad Prism version 5.00 (GraphPad Software, San Diego, CA). For plasma creatinine levels and quantification of the PAS-positive area, the Mann-Whitney $U$ test was used to compare different groups. For all other experiments, one-way analysis of variance followed by Tukey's multiple comparisons test was performed. Significant differences were considered when $P<0.05$.

\section{Results}

Effect of CIS on Body and Kidney Weights. The body weight of control rats significantly increased ( 17\%) during the experiment. However, this increase in body weight was marginal in animals that received CIS with and without intervention $(\sim 5 \%)$. The relative kidney weights for all CIS groups including those receiving intervention were significantly higher than control animals, which is indicative of CIS-induced renal damage (Supplemental Tables 1 and 2).

Renal Function and Histologic Findings. CIS controls showed a significant rise in plasma creatinine levels compared with the negative control group. A significant reduction was observed in creatinine levels in rats treated with EA or UA (Fig. 1A). Specifically, kidney histologic examination revealed degeneration of the tubules leading to cell detachment toward the tubular lumen in CIS controls, and such changes were not observed in the intervention groups (Fig. 1B). The morphologic tubular damage was further confirmed by PAS staining showing epithelial necrosis reflected by loss of brush border membrane, prominent PAS staining in tubular cells located toward the lumen of the tubules, and significant accumulation of PAS-positive material in the medullary region of the kidneys in the CIS controls (Fig. 2A). Animals treated with EA showed moderate protection, while UA offered significant protection (Fig. 2B).

Immunologic and Anti-Inflammatory Findings. Renal expression of TIM-1 was found to be significantly decreased in rats treated with EA and UA (Fig. 3, A-C). Additionally, the immunohistochemical staining for transcription factor $\mathrm{NF}-\kappa \mathrm{B}$ was prominent in cells detached from renal tubules and localized in the lumen, as can be observed in the CIS control kidney sections shown in Fig. 4A. In contrast,

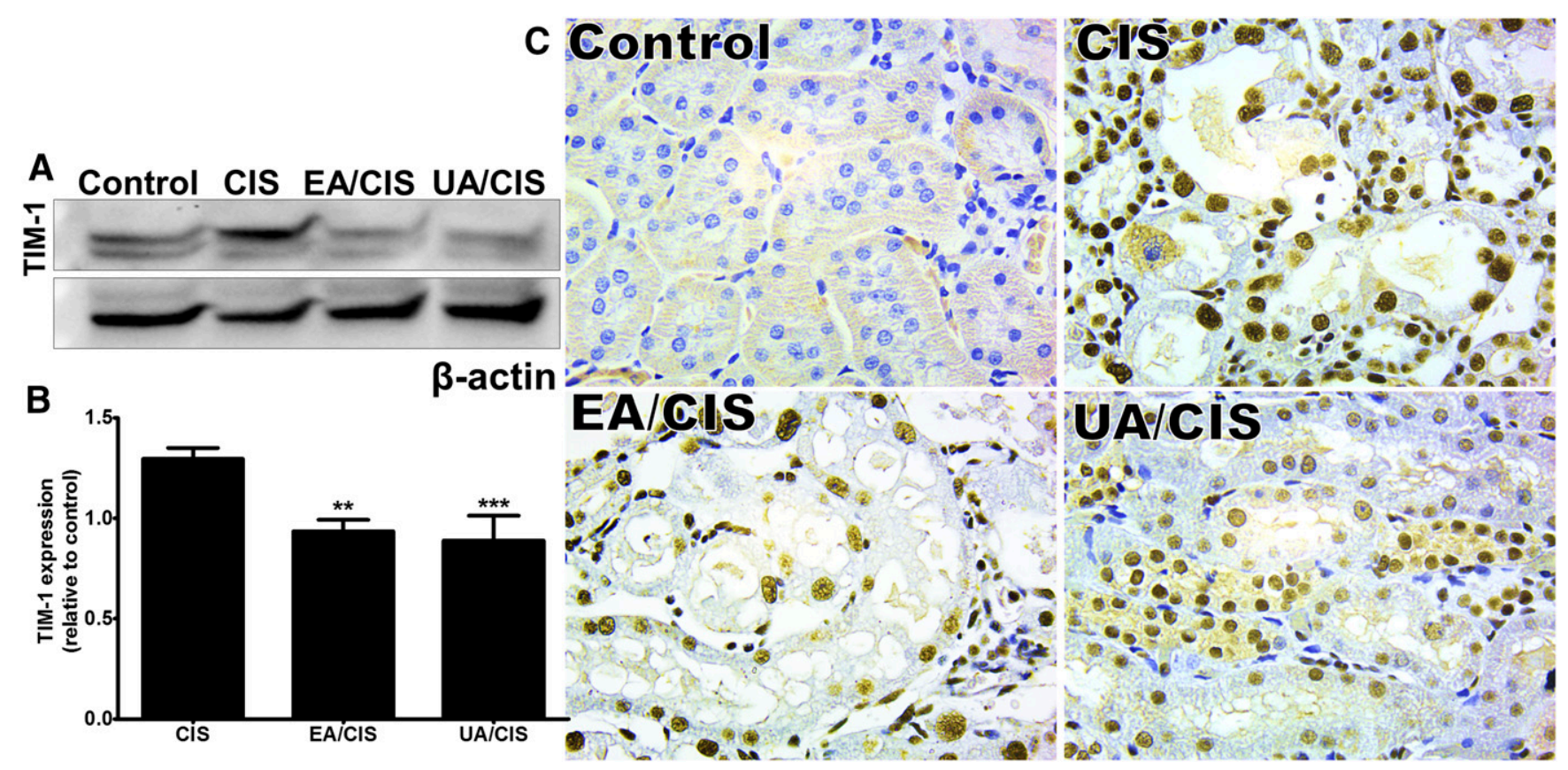

Fig. 3. UA and EA regulate TIM-1 protein expression in CIS-induced nephrotoxicity. (A) Representative image of western blot of TIM-1 expression in kidney homogenates for each group $(n=6)$ on day 6 after CIS administration to rats. (B) Relative band intensity analysis of TIM-1 expression of western blots normalized by $\beta$-actin. (C) Immunohistochemical staining of TIM-1 in kidney sections (golden brown-colored areas indicate positive staining; original magnification, $40 \times)$. $* * P<0.01$ and ${ }^{* * *} P<0.001$ compared with the CIS group; one-way analysis of variance followed by Tukey's multiple comparisons test. 


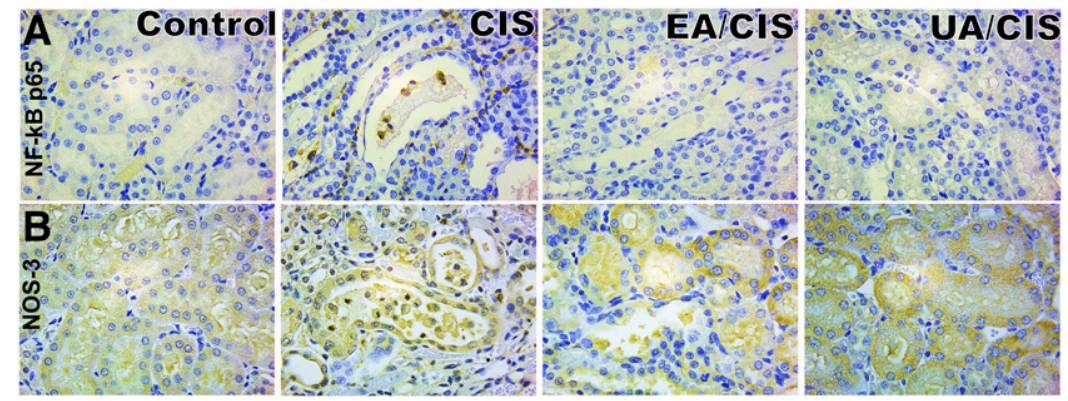

Fig. 4. Anti-inflammatory and prosurvival effects of UA and EA in CIS-induced kidney damage. Representative images of immunohistochemical staining of NF- $\kappa \mathrm{B}$ p65 (A) and NOS3 (B) in kidney sections for each group $(n=6)$ on day 6 after CIS administration to rats (golden browncolored areas indicate positive staining). Images were captured using a bright-field microscope at original magnification, $40 \times$

intervention groups displayed decreased staining in kidney sections, suggesting the prevention of NF- $\kappa \mathrm{B}$ expression (Fig. 4A). The expression of NOS3 was preserved in renal tubules in the intervention groups, while a decrease in the expression of the enzyme was observed in the kidneys of the CIS controls (Fig. 4B). Furthermore, the immunohistochemical analysis for Iba1, a protein specifically expressed in macrophages, showed marked staining in the renal tubular cells of the CIS control animals, indicating enhanced macrophage infiltration in the damaged tissue (Fig. 5A).
Interestingly, rats treated with UA had dramatically reduced Iba1 expression at the tubular region of the kidney, which was almost comparable to the negative control animals, while EA failed to attenuate (Fig. 5A). These findings further correlated with the expression of various inflammatory cytokines in kidney homogenates (Fig. 5, B-J; Supplemental Fig. 1). Specifically, UA treatment decreased the expression of proinflammatory cytokines, such as tumor necrosis factor- $\alpha$, interleukin (IL)-6, interferon gamma, $\mathrm{IL}-1 \alpha, \mathrm{IL}-1 \beta, \mathrm{IL}-13, \mathrm{IL}-17 \mathrm{~A}$, and IL-2. In contrast, EA treatment
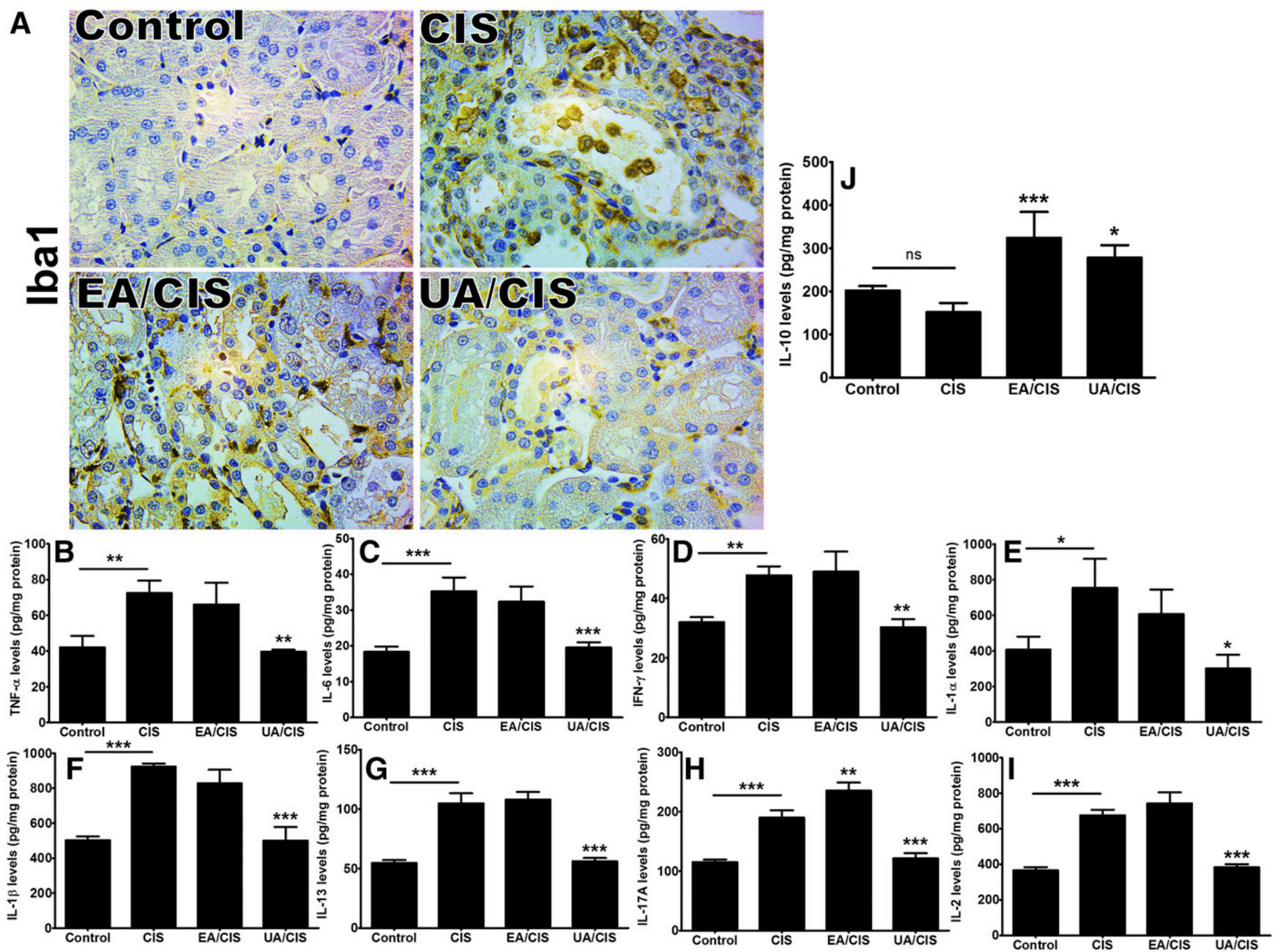

Fig. 5. Effect of UA and EA on macrophage infiltration and modulation of inflammatory cytokines in CIS-induced nephrotoxicity. (A) Representative image of immunohistochemical staining of Iba1 in kidney sections (golden brown-colored areas indicate positive staining; original magnification, $40 \times$ ). (B-J) Cytokine levels in kidney homogenates for each group $(n=6)$ on day 6 after CIS administration to rats. $* P<0.05, * * P<0.01$, and $* * * P<0.001$ compared with the CIS group; one-way analysis of variance followed by Tukey's multiple comparisons test. 
failed to reduce these levels (Fig. 5, B-I). Furthermore, both UA and EA treatments showed a marked increase in the levels of antiinflammatory cytokine IL-10 (Fig. 5J). These changes in the cytokine levels were localized in kidney tissue and no systemic inflammatory response was observed in plasma samples reflected by the absence of these cytokines.

Apoptosis Evaluation. As shown in Fig. 6A, a significant increase in the number of apoptotic cells localized in the tubular area was found in CIS control kidneys. In contrast, treatment with UA and EA considerably decreased the tubular apoptotic cells in the kidney sections Fig. 6B, suggesting the antiapoptotic activity of these compounds in tubular injury. Overall, UA offered better protection compared with EA in preventing CIS-induced nephrotoxicity by inhibiting inflammation and apoptosis.

\section{Discussion}

Since its first licensed use in 1978, CIS has been widely established in the treatment of various cancers. However, to date there has been limited advance in addressing the concerns related to CIS-induced nephrotoxicity (Loehrer and Einhorn, 1984). Alternative safer platinum-based analogs were developed but they were not as effective as CIS (Ho et al., 2016). The pathogenesis of CIS nephrotoxicity is characterized by renal dysfunction based on a rise in creatinine levels in plasma due to a decrease in the glomerular filtration rate, and is attributed to different mechanisms involving proapoptotic signaling, oxidative stress, and inflammation (Ramesh and Reeves, 2002; Hanigan and Devarajan, 2003; Yao et al., 2007). Overcoming inflammatory response through effective therapeutic agents is considered a significant approach to prevent nephrotoxicity (Ramesh and
Reeves, 2002; Pabla and Dong, 2008). However, to date there is no clear consensus on the therapeutic management of CIS-induced nephrotoxicity (Weijl et al., 2004; Baek et al., 2015; Yamamoto et al., 2016).

The dose of CIS used to induce renal damage in this study ( $5 \mathrm{mg} / \mathrm{kg}$ ) was selected based on previous literature (El-Garhy et al., 2014; Kursunluoglu et al., 2014). The doses of UA and EA were selected based on prior studies (Espín et al., 2013; García-Niño and Zazueta, 2015) as well as keeping in mind the translational feasibility of conventional dosage forms for oral administration ( $50 \mathrm{mg} / \mathrm{kg}$, human equivalent dose: $520 \mathrm{mg}$ for $65 \mathrm{~kg}$ person) (Food and Drug Administration, 2005).

The histologic and biochemical markers studied are reliable indicators in the experimental model (Nematbakhsh et al., 2013), which is also evident in our CIS-treated animals. UA, and to a lesser extent EA, improved renal function along with kidney morphology by attenuating the CIS-induced nephrotoxicity, which is localized in tubules and reflected by severe loss of brush borders membrane, detachment of cells toward the lumen of the tubules, and also prominent accumulation of PAS-positive material in the site of the renal injury.

TIM-1, also known as kidney injury molecule-1, is a protein markedly expressed by proximal tubular cells in the injured kidney. It has been proposed as a potential biomarker to detect and predict acute tubular injury in different stages of drug development and patient care since this protein is also found in urine and is stable over a prolonged period of time (Vaidya et al., 2010). TIM-1 signaling has been involved in promoting CIS nephrotoxicity by $\mathrm{T}$ cell activation and cytokine secretion and its inhibition is demonstrated to be highly protective (Nozaki et al., 2011). In our findings, we showed that the administration of UA or its precursor, EA, was able to suppress the renal protein expression of TIM-1, suggesting it as a potential target.
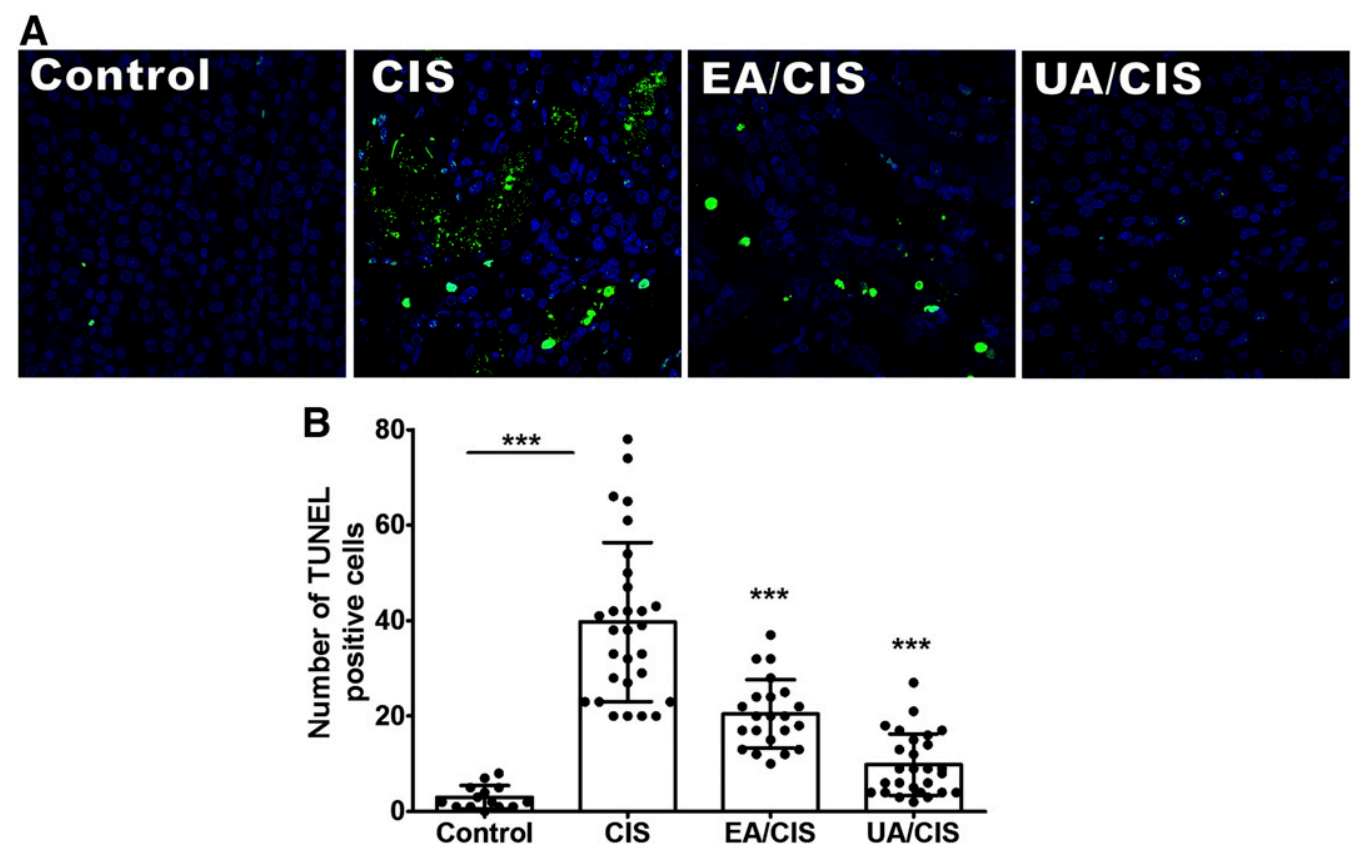

Fig. 6. Protective effect of UA and EA on tubular cell apoptosis in CIS-induced kidney injury. (A) Apoptotic cells were determined by terminal deoxynucleotidyl transferase dUTP nick end labeling (TUNEL) (green); nuclei were visualized by $4^{\prime}, 6$-diamidino-2-phenylindole staining (shown in blue) from each group $(n=6)$ on day 6 after CIS administration to rats. Representative images are shown at original magnification, $40 \times$. (B) Quantification of TUNEL-positive cells. ${ }^{* * *} P<0.001$ compared with the CIS group; one-way analysis of variance followed by Tukey's multiple comparisons test. 
The transcription factor NF- $\kappa \mathrm{B}$ has been implicated in the pathogenesis of CIS as a regulator of inflammation. The oxidative stress generated by this drug in renal cells activates the NF- $\kappa \mathrm{B}$ pathway, leading to stimulation of synthesis of many proinflammatory mediators (Schrier, 2002; Ozkok and Edelstein, 2014). Interestingly, both interventions, UA and EA, showed marked reduction of NF- $\kappa \mathrm{B}$ signaling in the renal tissue; however, only UA was also able to downregulate various cytokines involved in the proinflammatory cascade and immune response. This observation demonstrated that UA specifically targeted the $\mathrm{NF}-\kappa \mathrm{B}-$ induced proinflammatory response pathway. Since macrophages are involved in the release of cytokines, such inhibitory activity of these mediators obtained with UA may be due to its capacity to reduce macrophage infiltration in the tubular area of the kidney in CIS-induced nephrotoxicity. Moreover, it has been suggested that the upregulation of endogenous anti-inflammatory cytokine IL-10 produced by dendritic cells protects the kidney against CIS damage and its contribution to suppressing proinflammatory cytokines (Tadagavadi and Reeves, 2010). Our observations showed that both UA and EA treatments led to significant increase in renal IL-10 levels, suggesting that these compounds may have a direct anti-inflammatory effect in the proinflammatory response produced by CIS in kidney tissue. Along with these findings, the interventions were also able to reduce the number of apoptotic cells in the renal tubules probably mediated by the inhibition of $\mathrm{NF}-\kappa \mathrm{B}$, which is involved in the cell survival pathway. Interestingly, this antiapoptotic effect was more evident in the case of UA, suggesting that $\mathrm{NF}-\kappa \mathrm{B}$ is a direct target, and through modulating this pathway it is able to attenuate the damage in CIS-induced nephrotoxicity.

NOS3, known as endothelial nitric oxide synthase, is highly expressed in renal vascular endothelium but is also expressed in the cortex and medulla of proximal tubules (Mount and Power, 2006). Its activity has been associated with the renal protective effect of ischemic preconditioning against the ischemia/reperfusion-induced acute renal failure in a mouse model (Yamasowa et al., 2005). Here, we demonstrate that CIS reduced the NOS3 expression in the kidney, which can be explained by a decrease in the renal blood flow likely to occur in early CIS-induced acute renal failure (Winston and Safirstein, 1985). Since the nitric oxide synthase system is responsible for nitric oxide production, which regulates sodium and water homeostasis in the kidney (Mount and Power, 2006), any alteration in this pathway contributes to worsening of the tubular damage. This finding is in agreement with previous studies that showed decreased expression of NOS3 in CISinduced acute kidney injury in rats, and such expression was enhanced by $\alpha$-lipoic acid (Bae et al., 2009) and vitamin $\mathrm{E}$ (Darwish et al., 2017), suggesting the beneficial role of this enzyme in CIS-induced nephrotoxicity. UA and EA were able to reverse the effect of CIS-induced NOS3 modulation.

In summary, our data support the hypothesis that UA is a better therapeutic agent than its precursor EA in mitigating CIS-induced nephrotoxicity. However, further studies are needed to understand the intricacies and interplay of the molecular mechanism proposed in this research work. Overall, UA demonstrates significant potential as a good adjuvant for renal protection in patients receiving nephrotoxic drugs, such as CIS.

\section{Acknowledgments}

We are grateful to Dr. Gus A. Wright, Department of Veterinary Pathobiology (VTPB), Texas A\&M University, for providing access to the Digital Imaging Core Facility; and Dr. Andrew Hillhouse, Texas A\&M Institute for Genome Sciences and Society, for providing access to the Bio-Plex 200 system.

\section{Authorship Contributions}

Participated in research design: Guada, Ganugula, Vadhanam, Majeti.

Conducted experiments: Guada, Ganugula.

Performed data analysis: Guada, Ganugula, Majeti.

Wrote or contributed to the writing of the manuscript: Guada, Ganugula, Vadhanam, Majeti.

\section{References}

Al-Kharusi N, Babiker HA, Al-Salam S, Waly MI, Nemmar A, Al-Lawati I, Yasin J, Beegam S, and Ali BH (2013) Ellagic acid protects against cisplatin-induced nephrotoxicity in rats: a dose-dependent study. Eur Rev Med Pharmacol Sci 17: 299-310.

Apps MG, Choi EH, and Wheate NJ (2015) The state-of-play and future of platinum drugs. Endocr Relat Cancer 22:R219-R233.

Atessahín A, Ceríbași AO, Yuce A, Bulmus O, and Cikim G (2007) Role of ellagic acid against cisplatin-induced nephrotoxicity and oxidative stress in rats. Basic Clin Pharmacol Toxicol 100:121-126.

Bae EH, Lee J, Ma SK, Kim IJ, Frøkiaer J, Nielsen S, Kim SY, and Kim SW (2009) $\alpha$-Lipoic acid prevents cisplatin-induced acute kidney injury in rats. Nephrol Dial Transplant 24:2692-2700.

Baek SH, Kim SH, Kim JW, Kim YJ, Lee KW, and Na KY (2015) Effects of a DPP4 inhibitor on cisplatin-induced acute kidney injury: study protocol for a randomized controlled trial. Trials 16:239.

Barabas K, Milner R, Lurie D, and Adin C (2008) Cisplatin: a review of toxicities and therapeutic applications. Vet Comp Oncol 6:1-18.

Bhat ZY, Cadnapaphornchai P, Ginsburg K, Sivagnanam M, Chopra S, Treadway CK, Lin HS, Yoo G, Sukari A, and Doshi MD (2015) Understanding the risk factors and long-term consequences of cisplatin-associated acute kidney injury: an observational cohort study. PLoS One 10:e0142225.

Boulikas T (2009) Clinical overview on Lipoplatin: a successful liposomal formulation of cisplatin. Expert Opin Investig Drugs 18:1197-1218.

Ciccolini J, Mercier C, Dahan L, and André N (2011) Integrating pharmacogenetics into gemcitabine dosing-time for a change? Nat Rev Clin Oncol 8:439-444.

Darwish MA, Abo-Youssef AM, Khalaf MM, Abo-Saif AA, Saleh IG, and Abdelghany TM (2017) Vitamin E mitigates cisplatin-induced nephrotoxicity due to reversal of oxidative/nitrosative stress, suppression of inflammation and reduction of total renal platinum accumulation. J Biochem Mol Toxicol 31:1-9.

Dilruba S and Kalayda GV (2016) Platinum-based drugs: past, present and future. Cancer Chemother Pharmacol 77:1103-1124.

El-Garhy AM, Abd El-Raouf OM, El-Sayeh BM, Fawzy HM, and Abdallah DM (2014) Ellagic acid antiinflammatory and antiapoptotic potential mediate renoprotection in cisplatin nephrotoxic rats. J Biochem Mol Toxicol 28:472-479.

Espín JC, Larrosa M, García-Conesa MT, and Tomás-Barberán F (2013) Biological significance of urolithins, the gut microbial ellagic acid-derived metabolites: the evidence so far. Evid Based Complement Alternat Med 2013:270418.

US Food and Drug Administration; USFDA (2005) Guidance for industry: estimating the maximum safe starting dose in initial clinical trials for therapeutics in adult healthy volunteers, Food and Drug Administration, Rockville, MD.

García-Niño WR and Zazueta C (2015) Ellagic acid: pharmacological activities and molecular mechanisms involved in liver protection. Pharmacol Res 97:84-103.

Hanigan MH and Devarajan P (2003) Cisplatin nephrotoxicity: molecular mechanisms. Cancer Ther 1:47-61.

Ho GY, Woodward N, and Coward JI (2016) Cisplatin versus carboplatin: comparative review of therapeutic management in solid malignancies. Crit Rev Oncol Hematol 102:37-46.

Kang I, Buckner T, Shay NF, Gu L, and Chung S (2016) Improvements in metabolic health with consumption of ellagic acid and subsequent conversion into urolithins: evidence and mechanisms. Adv Nutr 7:961-972.

Koyner JL, Sher Ali R, and Murray PT (2008) Antioxidants. Do they have a place in the prevention or therapy of acute kidney injury? Nephron, Exp Nephrol 109:e109-e117.

Kursunluoglu G, Kayali HA, and Taskiran D (2014) The effect of cisplatin toxicity and capsaicin on electron transport chain in liver and kidney of Sprague Dawley rats. Cell Biochem Biophys 69:707-716.

Lecumberri E, Dupertuis YM, Miralbell R, and Pichard C (2013) Green tea polyphenol epigallocatechin-3-gallate (EGCG) as adjuvant in cancer therapy. Clin Nutr 32:894-903.

Liberal J, Carmo A, Gomes C, Cruz MT, and Batista MT (2017) Urolithins impair cell proliferation, arrest the cell cycle and induce apoptosis in UMUC3 bladder cancer cells. Invest New Drugs, 1-11.

Loehrer PJ and Einhorn LH (1984) Drugs five years later. Cisplatin. Ann Intern Med 100:704-713.

Mathijssen RH, Sparreboom A, and Verweij J (2014) Determining the optimal dose in the development of anticancer agents. Nat Rev Clin Oncol 11:272-281.

Mount PF and Power DA (2006) Nitric oxide in the kidney: functions and regulation of synthesis. Acta Physiol (Oxf) 187:433-446. 
Nematbakhsh M, Ashrafi F, Nasri H, Talebi A, Pezeshki Z, Eshraghi F, and Haghighi M (2013) A model for prediction of cisplatin induced nephrotoxicity by kidney weight in experimental rats. J Res Med Sci 18:370-373.

Nozaki Y, Nikolic-Paterson DJ, Yagita H, Akiba H, Holdsworth SR, and Kitching AR (2011) Tim-1 promotes cisplatin nephrotoxicity. Am J Physiol Renal Physiol 301: F1098-F1104.

Ozkok A and Edelstein CL (2014) Pathophysiology of cisplatin-induced acute kidney injury. BioMed Res Int 2014:967826.

Pabla N and Dong Z (2008) Cisplatin nephrotoxicity: mechanisms and renoprotective strategies. Kidney Int 73:994-1007.

Ramesh G and Reeves WB (2002) TNF- $\alpha$ mediates chemokine and cytokine expression and renal injury in cisplatin nephrotoxicity. J Clin Invest 110:835-842.

Ryu D, Mouchiroud L, Andreux PA, Katsyuba E, Moullan N, Nicolet-Dit-Félix AA, Williams EG, Jha P, Lo Sasso G, Huzard D, et al. (2016) Urolithin A induces mitophagy and prolongs lifespan in $C$. elegans and increases muscle function in rodents. Nat Med 22:879-888.

Saha P, Yeoh BS, Singh R, Chandrasekar B, Vemula PK, Haribabu B, VijayKumar M, and Jala VR (2016) Gut microbiota conversion of dietary ellagic acid into bioactive phytoceutical urolithin A inhibits heme peroxidases. PLoS One 11:e0156811.

Schrier RW (2002) Cancer therapy and renal injury. J Clin Invest 110:743-745.

Sharma G, Italia JL, Sonaje K, Tikoo K, and Ravi Kumar MN (2007) Biodegradable in situ gelling system for subcutaneous administration of ellagic acid and ellagic acid loaded nanoparticles: evaluation of their antioxidant potential against cyclosporine induced nephrotoxicity in rats. $J$ Control Release 118:27-37.

Sonaje K, Italia JL, Sharma G, Bhardwaj V, Tikoo K, and Kumar MN (2007) Development of biodegradable nanoparticles for oral delivery of ellagic acid and evaluation of their antioxidant efficacy against cyclosporine A-induced nephrotoxicity in rats. Pharm Res 24:899-908.

Tadagavadi RK and Reeves WB (2010) Endogenous IL-10 attenuates cisplatin nephrotoxicity: role of dendritic cells. J Immunol 185:4904-4911.

Tang L, Mo Y, Li Y, Zhong Y, He S, Zhang Y, Tang Y, Fu S, Wang X, and Chen A (2017) Urolithin A alleviates myocardial ischemia/reperfusion injury via PI3K/Akt pathway. Biochem Biophys Res Commun 486:774-780.
Vaidya VS, Ozer JS, Dieterle F, Collings FB, Ramirez V, Troth S, Muniappa N, Thudium D, Gerhold D, Holder DJ, et al. (2010) Kidney injury molecule-1 outperforms traditional biomarkers of kidney injury in preclinical biomarker qualification studies. Nat Biotechnol 28:478-485.

Weijl NI, Elsendoorn TJ, Lentjes EG, Hopman GD, Wipkink-Bakker A, Zwinderman $\mathrm{AH}$, Cleton FJ, and Osanto S (2004) Supplementation with antioxidant micronutrients and chemotherapy-induced toxicity in cancer patients treated with cisplatin-based chemotherapy: a randomised, double-blind, placebo-controlled study. Eur J Cancer 40:1713-1723.

Winston JA and Safirstein R (1985) Reduced renal blood flow in early cisplatininduced acute renal failure in the rat. Am J Physiol 249:F490-F496.

Yamamoto Y, Watanabe K, Tsukiyama I, Yabushita H, Matsuura K, and Wakatsuki A (2016) Hydration with $15 \mathrm{mEq}$ magnesium is effective at reducing the risk for cisplatin-induced nephrotoxicity in patients receiving cisplatin $\left(\geq 50 \mathrm{mg} / \mathrm{m}^{2}\right) \mathrm{com}$ bination chemotherapy. Anticancer Res 36:1873-1877.

Yamasowa H, Shimizu S, Inoue T, Takaoka M, and Matsumura Y (2005) Endothelial nitric oxide contributes to the renal protective effects of ischemic preconditioning. $J$ Pharmacol Exp Ther 312:153-159.

Yao X, Panichpisal K, Kurtzman N, and Nugent K (2007) Cisplatin nephrotoxicity: a review. Am J Med Sci 334:115-124.

Yuan T, Ma H, Liu W, Niesen DB, Shah N, Crews R, Rose KN, Vattem DA and Seeram NP (2016) Pomegranate's neuroprotective effects against Alzheimer's Disease are mediated by urolithins, its ellagitannin-gut microbial derived metabolites. ACS Chem Neurosci 7:26-33.

Zhou B, Wang J, Zheng G, and Qiu Z (2016) Methylated urolithin A, the modified ellagitannin-derived metabolite, suppresses cell viability of DU145 human prostate cancer cells via targeting miR-21. Food Chem Toxicol 97:375-384.

Address correspondence to: Majeti N.V. Ravi Kumar, Department of Pharmaceutical Sciences, College of Pharmacy, Texas A\&M University, Reynolds Medical Bldg., Mail Stop 1114, College Station, TX 77843. E-mail: mnvrkumar@tamhsc.edu 